\section{CASE REPORT}

\section{A.T. Karagulle Kendi}

C. Krenzel

F.W. Ott

J.R. Brace

S.K. Norberg

S.A. Kieffer

\title{
Poststreptococcal Dystonia with Bilateral Striatal Enlargement: MR Imaging and Spectroscopic Findings
}

SUMMARY: Isolated bilateral striatal necrosis is an abnormality of the basal ganglia associated with acute dystonia in children. This report describes the development of dystonic movements in a 7-year-old male patient 2 weeks after streptococcal pharyngitis.

D ystonia with basal ganglial imaging abnormalities is usually related to structural, metabolic, toxic, traumatic, or vascular disorders. ${ }^{1,2}$ Swedo et $\mathrm{al}^{3}$ coined the term "pediatric autoimmune neuropsychiatric disorders associated with streptococcal infection" (PANDAS) to describe them. MR imaging in patients with suspected PANDAS has demonstrated a lack of structural abnormality, though volumetric evaluations revealed enlargement of the basal ganglia. ${ }^{4}$

This report describes a patient presenting with dystonia and basal ganglial imaging abnormalities following streptococcal infection. Because there is no specific test to confirm this diagnosis, poststreptococcal dystonia was presumptive. To our knowledge, this is the first case report addressing the findings on diffusion-weighted MR imaging and on MR spectroscopy in a patient with poststreptococcal dystonia and striatal enlargement.

\section{Case Report}

A 7-year-old boy presented with rigidity and difficulty breathing. Diagnosis of streptococcal pharyngitis was made, and he was treated with an aminopenicillin. Two days later, he was unable to get out of bed, and persistent drooling was noted. Dexamethasone was begun, but his condition deteriorated with increasing rigidity, dystonia, drooling, and tremor of the hands. Rigidity and dystonia rapidly progressed, and he developed mutism, dysphagia, and incontinence. Laboratory testing demonstrated borderline high antistreptolysin $\mathrm{O}$ titer and a high level of antideoxyribonuclease antibody.

Cranial MR imaging demonstrated bilateral T2 hyperintensity in the heads of the caudate nuclei, the putamina, and the external/ extreme capsules. The heads of the caudate nuclei and the putamina were enlarged (Fig 1) and demonstrated minimal restricted diffusion on diffusion-weighted images (Fig 2). Single-voxel MR spectroscopy of the left lentiform nucleus demonstrated elevation of the lipid/lactate peak and a decreased $N$-acetylaspartate (NAA) peak (Fig $3 A$ ).

Follow-up MR imaging 1 month later showed no significant

Received December 3, 2007; accepted after revision January 9, 2008.

From the Division of Neuroradiology (A.T.K.K., C.K., F.W.O., J.R.B., S.A.K.), Department of Radiology, University of Minnesota Medical School, Minneapolis, Minn; and Division of Pediatric Neurology (S.K.N.), Gillette Children's Specialty Healthcare, St. Paul, Minn.

Please address correspondence to A. Tuba Karagulle Kendi, MD, University of Minnesota, Department of Radiology, MMC 292 Mayo, 8292, 420 Delaware, Minneapolis, MN 55455; e-mail: drtubakendi@yahoo.com

DOI 10.3174/ajnr.A1065

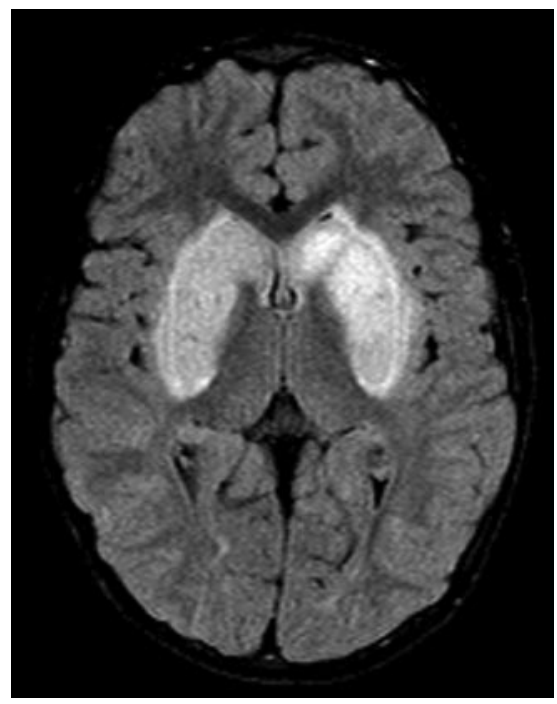

Fig 1. Axial turbo fluid-attenuated inversion recovery image demonstrates bilateral $\mathrm{T} 2$ hyperintensity within the heads of the caudate nuclei, the putamina, and the external/ extreme capsules. The heads of the caudate/putamina are enlarged.

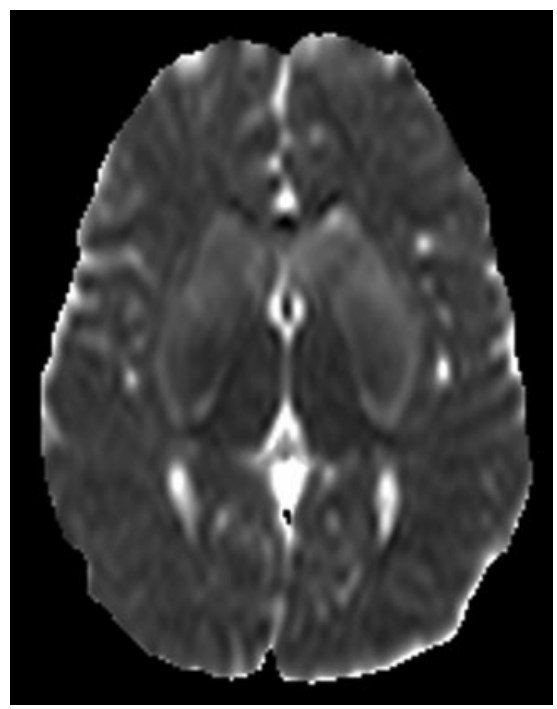

Fig 2. Axial apparent diffusion coefficent map shows restricted diffusion in the heads of the caudate and the putamina bilaterally.

change. MR spectroscopy demonstrated continued depression of the NAA peak with interval increase in the choline/creatine ratio compared with the initial MR spectroscopy (Fig 3B). 

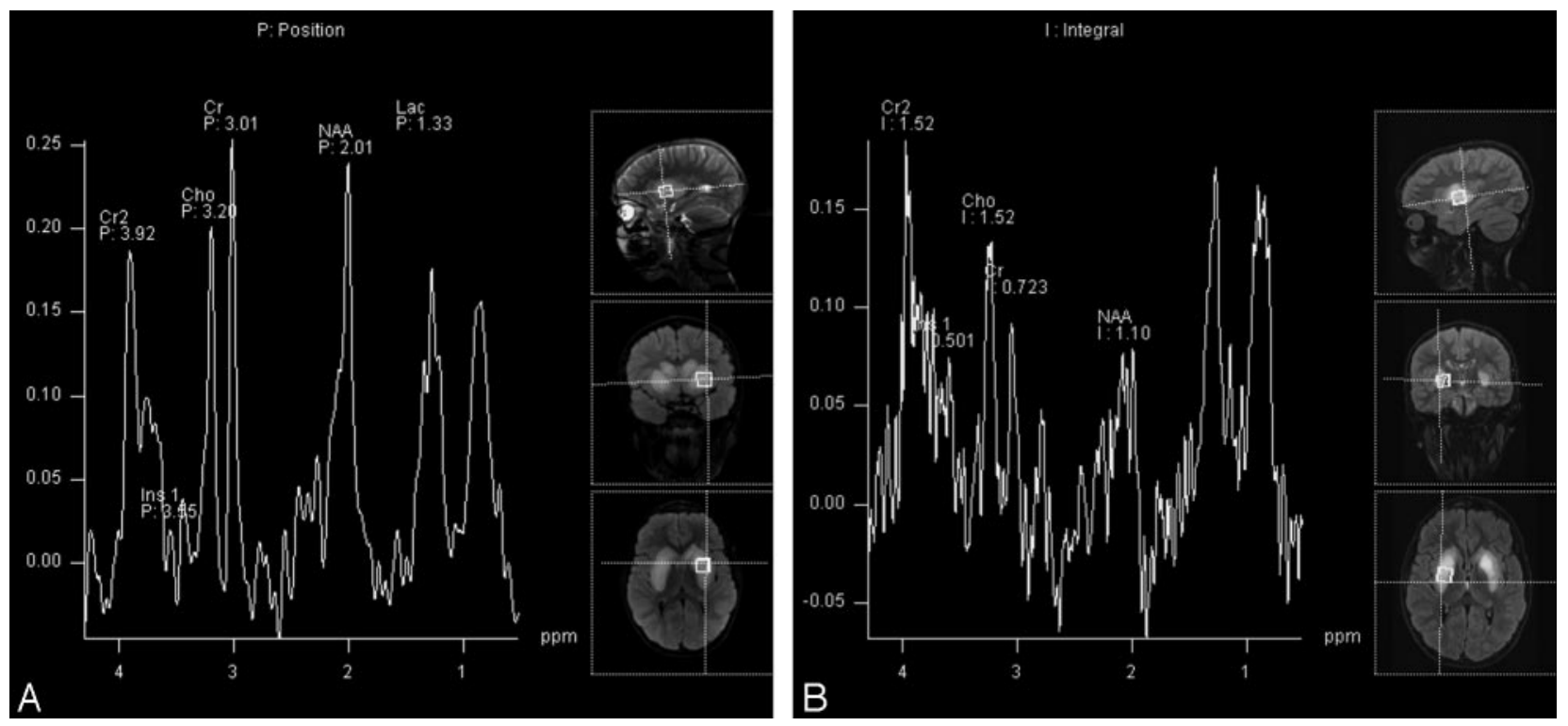

Fig 3. $A$, Initial single-voxel MR spectroscopy of the left putamen demonstrates an elevated lipid/lactate (Lac) peak and a decreased NAA peak. $B$, Follow-up MR spectroscopy of the right putamen shows further decrease in the NAA level with an increase in the choline (Cho)/creatine (Cr) ratio. Ins indicates myo-inositol
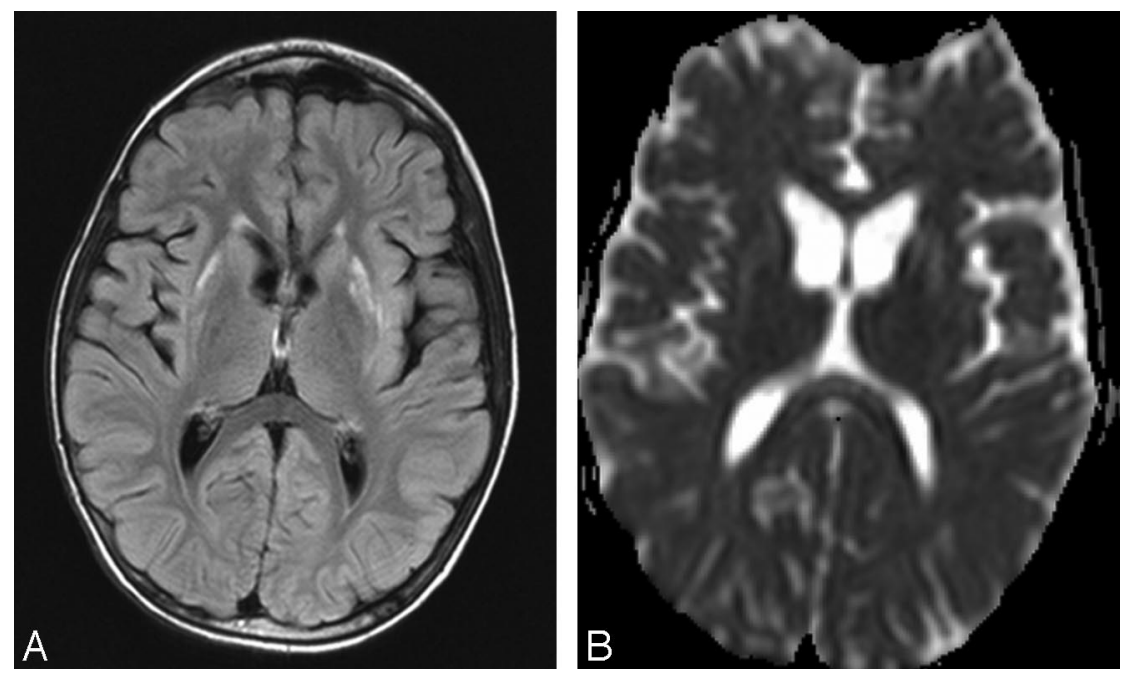

Fig 4. A, Axial fluid-attenuated inversion recovery image demonstrates only a small rim of residual T2 hyperintensity at the lateral margin of the right putamen. Slightly greater T2 hyperintensity remains in the left putamen. B, Axial apparent diffusion coefficient map demonstrates complete resolution of restricted diffusion in the heads of the caudate nuclei and putamina bilaterally.

At the time of discharge, the patient exhibited mild improvement in neurologic functions. He participated in aggressive physical therapy, and 12-month follow-up demonstrated significant overall clinical improvement. Twelve-month follow-up MR imaging revealed significant improvement in the size and the extent of T2 hyperintensity in the heads of the caudate nuclei, the putamina, and the external/extreme capsules. There is now residual abnormal T2 hyperintensity only in the lateral aspects of both putamina. Enlargement of the heads of the caudate nuclei and putamina has completely resolved (Fig $4 A$ ), and no restricted diffusion is apparent in these structures (Fig 4B).

\section{Discussion}

Although the classic poststreptococcal neuropsychiatric disorder is Sydenham chorea, recently other poststreptococcal basal ganglia phenotypes have been described in patients who do not meet the criteria for Sydenham chorea. One of these disorders is poststreptococcal dystonia. ${ }^{5}$ There is evidence suggesting that these disorders are autoimmune-mediated with antibodies attacking certain parts of the brain, specifically the basal ganglia. ${ }^{5}$

A variety of MR imaging findings have been reported in patients with Sydenham chorea, varying from normal-to-abnormal signal intensity involving the basal ganglia. ${ }^{6-9}$ Dale et $\mathrm{al}^{2}$ described 2 children with poststreptococcal dystonia. The putamina and the heads of the caudate nuclei demonstrated T2 hyperintensity, but no mention was made regarding the size of these nuclei.

MR spectroscopic studies of the basal ganglia in children with Sydenham chorea and rheumatic fever have revealed reduction in NAA, increase in the choline/creatine ratio, and abnormal peaks at 3.5-4.2 $\mathrm{ppm}^{6}$ and at $1.5 \mathrm{ppm}^{7}$ Because choline is involved in neuronal and glial phospholipid metabolism, an elevated choline/creatine ratio may imply disturbed cell membrane turnover, whereas a decreased NAA/creatine ratio may indicate neuronal dysfunction or loss. ${ }^{6}$ An increased choline/creatine ratio may also reflect imbalance between dopaminergic and cholinergic systems because an increased cho- 
line/creatine ratio has been found in the basal ganglia of patients with Parkinson disease and attention deficit/hyperactivity disorder. ${ }^{10}$ The peaks at 3.5-4.2 and $1.5 \mathrm{ppm}$ are thought to reflect breakdown products of lipids and amino acids. ${ }^{6,7}$ Carbohydrates also resonate between 3.5-5 ppm. This supports the presence of antibodies attacking the basal ganglia because antibodies against streptolysin $\mathrm{O}$ are known to be mostly immunoglobulin $\mathrm{M}$ type, and they are composed of $15 \%$ carbohydrates. $^{7}$

Single-voxel MR spectroscopy of the abnormal basal ganglia in our patient showed reduction of the NAA peak (a sign of neuronal injury) and an increased lactate peak, corresponding to conversion from aerobic energy production to anaerobic glycolysis. ${ }^{11}$ Follow-up MR spectroscopy 1 month after the initial examination demonstrated further decrease in NAA and interval increase in choline with reversal of the choline/ creatine ratio, consistent with increased neuronal injury and increased cell membrane turnover. In other pathologies marked by cell membrane breakdown (eg, neurodegenerative disorders), bound moieties may be liberated into the free choline pool, contributing to an increase in choline resonance. ${ }^{6}$ Recent studies by using immunofluorescence documented serum anti-basal ganglial antibodies in the poststreptococcal basal ganglia. ${ }^{6} \mathrm{MR}$ spectroscopy findings in our patient support the hypothesis that the pathophysiologic mechanism for poststreptococcal dystonia with striatal necrosis could be an antibody-mediated immune reaction.

Follow-up MR imaging at 1 year demonstrated interval improvement in size, T2 signal intensity, and diffusion of the basal ganglia and appears to correlate with a slowly improving clinical course. These findings suggest that the underlying pathology in isolated basal ganglial "necrosis" may not represent irreversible necrosis after all.

\section{References}

1. Goutieres F, Aicardi J. Acute neurological dysfunction associated with destructive lesions of the basal ganglia in children. Ann Neurol 1982;12:328-32

2. Dale RC, Church AJ, Surtees RA, et al. Post-streptococcal autoimmune dystonia with isolated bilateral striatal necrosis. Dev Med Child Neurol 2002;44:485-89

3. Swedo SE, Leonard HL, Garvey M, et al. Pediatric autoimmune neuropsychiatric disorders associated with streptococcal infections: clinical description of the first 50 cases. Am J Psychiatry 1998;155:264-71

4. Giedd JN, Rapoport JL, Garvey MA, et al. MRI assessment of children with obsessive compulsive disorder or tics associated with streptococcal infection. Am J Psychiatry 2000;157:281-83

5. Dale RC, Church AJ, Surtees RAH, et al. Post-streptococcal autoimmune neuropsychiatric disease presenting as paroxysmal dystonic choreoathetosis. Mov Disord 2002;17:817-20

6. Margari L, Ventura P, Portoghese C, et al. Brain magnetic resonance spectroscopy in Sydenham's chorea and ADHD. Pediatric Neurol 2006;34:467-73

7. Castillo M, Kwock L, Arbelaez A. Syndenham's chorea: MRI and proton spectroscopy. Neuroradiology 1999;41:943-45

8. Emery ES, Vieco PT. Sydenham chorea: magnetic resonance imaging reveals permanent basal ganglia injury. Neurology 1997;48:531-33

9. Traill Z, Pike M, Byrne J. Sydenham's chorea: a case showing reversible striatal abnormalities on CT and MRI. Dev Med Child Neurol 1995;37:270-73

10. Jin Z, Zang YF, Zeng YW, et al. Striatal neuronal loss or dysfunction and choline rise in children with attention-deficit hyperactivity disorder: a $1 \mathrm{H}$ magnetic resonance spectroscopy study. Neurosci Lett 2001;23:45-48

11. Michel SJ, Given CA, Robertson WC. Imaging of the brain, including diffusion-weighted imaging in methylmalonic acidemia. Pediatr Radiol 2004;34: 580-82. Epub 2004 Mar 9 